\title{
Estudo comparativo da estabilidade global em diferentes tipos de lajes
}

A verificação da estabilidade global em edifícios de concreto armado é um parâmetro bastante complexo no que tange a resistência e durabilidade da estrutura. Neste trabalho, analisou-se um modelo estrutural para três tipos de lajes, modeladas pelo software AltoQi Eberick v10. O primeiro modelo considera o pórtico com laje maciça. O segundo considera o edifício com laje nervurada e o terceiro, com laje treliçada. Os resultados a serem considerados são o coeficiente Gama-Z e rigidez lateral. Essas análises foram comparadas, e observou-se uma diferença na distribuição das cargas para as vigas, os deslocamentos e, consequentemente, a estabilidade do Edifício, percebeu-se também que os valores de Gama-Z não alteraram de forma significativa, mostrando que as formações de pórtico têm a maior contribuição. Desse modo, os resultados obtidos comprovaram a importância de um estudo comparativo entre as lajes antes da elaboração de projetos estruturais, ressaltado também, é claro, o custo-benefício.

Palavras-chave: Coeficiente Gama-Z; Deslocamentos; Rigidez Lateral.

\section{Comparative study of global stability in different types of slabs}

The verification of the overall stability in reinforced concrete buildings is a very complex parameter regarding the strength and durability of the structure. In this work, we analyzed a structural model for three types of slabs, modeled by the AltoQi Eberick v10 software. The first model considers the massive slab portico. The second considers the building with ribbed slab and the third model with lattice slab. The results to be considered are the Gamma- $Z$ coefficient and lateral stiffness. These analyzes were compared, and there was a difference in the load distribution to the beams, the displacements and consequently the stability of the building. It was also noticed that the Z-Gamma values did not change significantly, showing that the gantry have the largest contribution. Thus, the results showed the importance of a comparative study between the slabs before the elaboration of structural projects, emphasizing the cost-benefit is also clear.

Keywords: Gamma-Z coefficient; Displacements; Lateral stiffness.

Topic: Engenharia Civil

Reviewed anonymously in the process of blind peer.
Received: 11/11/2019

Approved: 21/12/2019
Murylo Rodrigues Cândido de Oliveira

Instituto Tocantinense Presidente Antônio Carlos, Brasil

http://lattes.cnpq.br/7522351934453711

muryloengcivil@gmail.com

\section{Marcos Vinicius Dias da Silva}

Instituto Tocantinense Presidente Antônio Carlos, Brasil http://lattes.cnpq.br/6838925859227163

viniciusmarcosdias12@gmail.com

\section{Mateus Borba Teixeira}

Instituto Tocantinense Presidente Antônio Carlos, Brasil

http://lattes.cnpq.br/7066869794283823

engmateusb@gmail.com
Douglas Freitas Augusto dos Santos

Instituto Tocantinense Presidente Antônio Carlos, Brasil

http://lattes.cnpq.br/6181125172616043

eng.prof.santos@gmail.com
Referencing this:

OLIVEIRA, M. R. C.; SILVA, M. V. D.; TEIXEIRA, M. B.; SANTOS, D. F. A. Estudo comparativo da estabilidade global em diferentes tipos de lajes. Technology Science, v.2, n.1, p.28-32, 2020. DOI: http://doi.org/10.6008/CBPC2674-6425.2020.001.0005 


\section{INTRODUÇÃO}

$\mathrm{Na}$ Engenharia Estrutural, sobretudo quando se refere a obras feitas de concreto armado, geralmente os sistemas estruturais são compostos por vigas, pilares e lajes. Os esforços, como se sabe, são transmitidos das lajes para as vigas, das vigas para os pilares e dos pilares para a fundação. Evidentemente trata-se de um processo complexo e que se constitui uma área com vários temas relevantes para serem estudados. No entanto, este trabalho limita-se apenas ao estudo das lajes. Conforme Pinheiro (2007) as lajes, de forma simples, podem ser definidas como sendo um elemento estrutural plano, em geral horizontal, representado como uma placa porque tem duas de suas dimensões (comprimento e largura) que são consideravelmente superiores à outra (espessura).

Ainda segundo Pinheiro (2007), os estudos nesta área, há cerca de trinta anos estavam pouco evoluídos. Os engenheiros que desejavam trabalhar com projetos de lajes pré-moldadas, por exemplo, encontravam dificuldades devido ao fato de não ter muita literatura sobre o tema para se orientar, ou seja, havia muito empirismo.

Todavia, nos dias de hoje, a engenharia civil se desenvolve de forma rápida e constante, o mercado está mais exigente tanto em relação à segurança e qualidade quanto em menor custo de execução das obras. Por isso, é imprescindível que se desenvolva materiais e métodos construtivos que garantam um melhor desempenho das edificações. Como foram desenvolvidos vários métodos construtivos, é necessário que se faça um estudo comparativo entre eles para se determinar qual é o mais indicado para uma determinada situação.

Logo, tem-se como principal propósito na realização deste trabalho o estudo, sempre baseado em ideias de alguns autores, do software AltoQi Eberick v10 e de acordo com as NBR's, sobre as lajes do tipo Maciças, Nervuradas e Treliçadas. Mais especificamente, realizou-se uma comparação do desempenho desses três métodos construtivos para, consequentemente, discorrer quais as vantagens e desvantagens de cada modelo.

\section{MATERIAIS E MÉTODOS}

O estudo será realizado em uma edificação hipotética, considerando a localização na cidade de Porto Nacional/TO. A edificação possui 15 metros de altura a parti do nível zero, possuindo o pavimento térreo e quatro pavimentos tipo com quatro salas comerciais (escritórios), com um banheiro em cada sala, conforme ilustrado na Figura 1.

O elemento da superestrutura foi modelado no software AltoQi Eberick v10, as vigas e pilares foram considerados como elementos de barras e a laje como elemento de casca, devido a sua rigidez os esforços laterais são transmitidos aos pilares. $\mathrm{O}$ concreto possui um $\mathrm{f}_{\mathrm{ck}}$ de $25 \mathrm{MPa}$ e módulo de elasticidade $\mathrm{E}=28000$ MPa, que foi calculado conforme a equação (1) da NBR 6118 (2014) mostrada a seguir.

$$
E_{c i}=\alpha e .5600 . \sqrt{ } f c k
$$




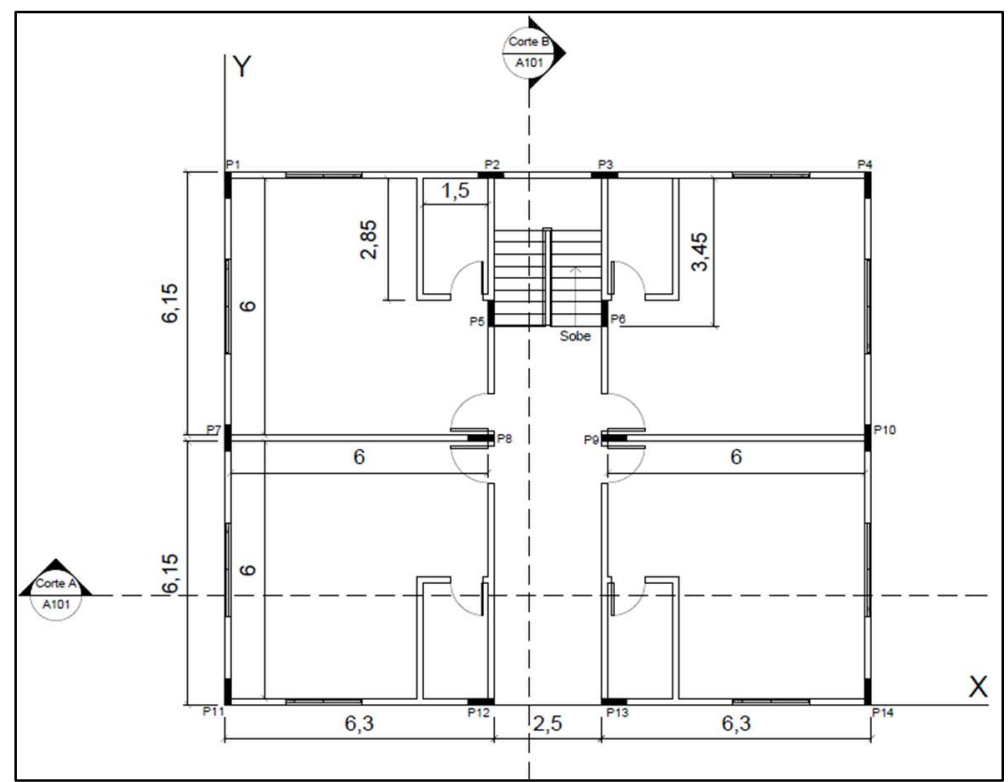

Figura 1: Planta Tipo.

As paredes externas e internas são constituídas por blocos de alvenaria de vedação possuindo $15 \mathrm{~cm}$ de espessura e a resistência característica em projeto do concreto das vigas, lajes e pilares é de $25 \mathrm{MPa}$. As ações do vento têm grande influência na estabilidade global da estrutura de acordo com as condições específicas da região onde será construída a edificação. Desse modo, para a verificação da velocidade característica do vento foi considerada a velocidade básica segundo o mapa das isopletas e conforme estabelecido na NBR 6123 (1988) um $\mathrm{V}_{\mathrm{o}}=30 \mathrm{~m} / \mathrm{s}$.

Com relação ao Fator Topográfico ( $\left.\mathrm{S}_{1}\right)$, segundo o item 5.2 da NBR 6123 (ABNT, 1988), foi considerado $S_{1}=1,0$ para terrenos planos ou pouco acidentados; em relação ao fator $\left(S_{2}\right)$, que é resultado da associação de fatores como rugosidade do terreno, dimensões do prédio e altura sobre o terreno, foi adotada classe $\mathrm{A}$ ('maior dimensão da estrutura não ultrapasse $20 \mathrm{~m}$ ') e categoria IV ('Terrenos cobertos por obstáculos numerosos e pouco espaçados, em zona florestal, industrial ou urbanizada') sendo obtido $F R=1, p=0,12$ e $b=0,86$.

Por fim, de acordo com a tabela 3, Valores mínimos do fator estatístico (S3) da NBR 6123 (1988), foi adotado $S_{3}=1,0$ para o edifício em estudo que se enquadra no grupo 2 (Edificações para hotéis e residências. Edificações para comércio e indústria com alto fator de ocupação). A partir da obtenção desses fatores foi possível calcular a velocidade característica do vento $(\mathrm{Vk})$, seguindo os procedimentos sugeridos pela NBR 6123 (ABNT, 1988) para a direção $x$ e $y$.

Para a verificação dos parâmetros de instabilidade da estrutura, foram analisados três tipos de lajes sendo elas maciça, nervurada e treliçada. O edifício foi modelado no software AltoQi Eberick v10 conforme ilustrado na figura 2. As análises foram realizadas em pórtico espacial e as lajes pelo método da analogia de grelha. 


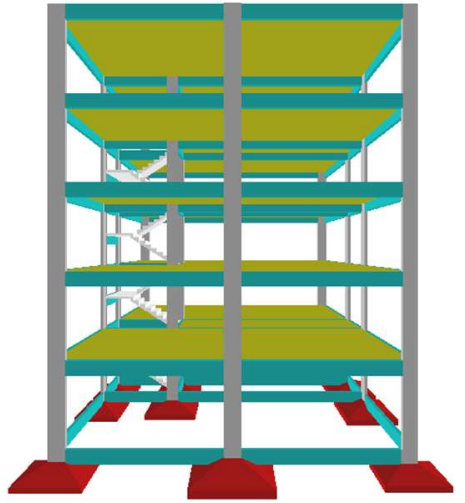

(a) Vista lateral esquerda.

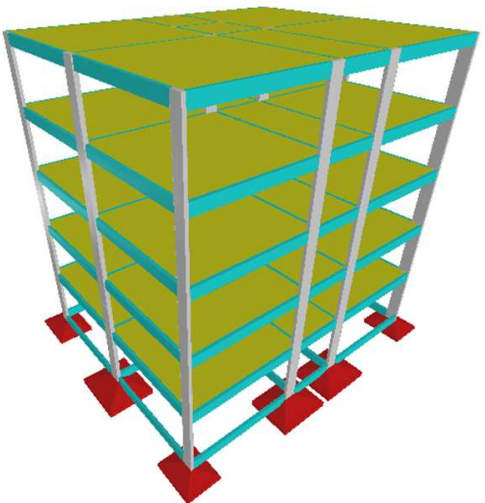

(b) Vista frontal.

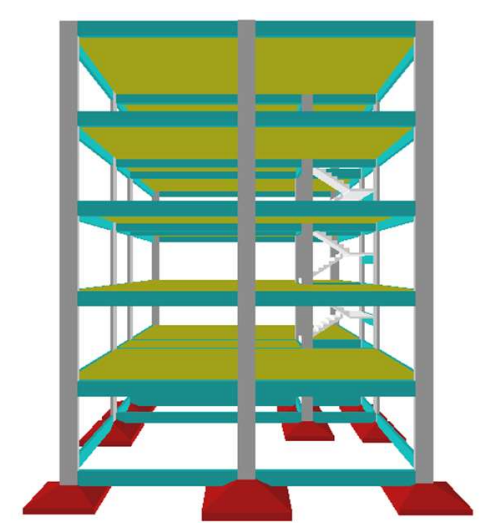

(c) Vista lateral direita.

Figura 2: Modelo do pórtico analisado.

\section{RESULTADOS E DISCUSSÃO}

Diante do que foi analisado no decorrer do trabalho, foi possível verificar os resultados das análises realizadas pelo software AltoQi Eberick v10 para os três tipos de lajes em estudo. Desse modo, o próprio programa gerou os resultados da estabilidade global, sendo apresentado na tabela 1 um comparativo entre os deslocamentos horizontais obtidos em cada estrutura.

Tabela 1: Comparação dos deslocamentos horizontais.

\begin{tabular}{|c|c|c|c|c|c|c|c|c|}
\hline \multicolumn{3}{|c|}{ Estrutura com laje maciça } & \multicolumn{3}{|c|}{ Estrutura com laje nervurada } & \multicolumn{3}{|c|}{ Estrutura com laje treliçada } \\
\hline \multicolumn{3}{|c|}{ Eixo $X(1.3 \mathrm{G} 1+1.4 \mathrm{G} 2+1.4 \mathrm{~S}+1.4 \mathrm{Q}+1.2 \mathrm{~A}+0.84 \mathrm{~V} 1)$} & \multicolumn{3}{|c|}{ Eixo $X(1.3 \mathrm{G} 1+1.4 \mathrm{G} 2+1.4 \mathrm{~S}+1.4 \mathrm{Q}+1.2 \mathrm{~A}+0.84 \mathrm{~V} 1)$} & \multicolumn{3}{|c|}{ Eixo $X(1.3 \mathrm{G} 1+1.4 \mathrm{G} 2+1.4 \mathrm{~S}+1.4 \mathrm{Q}+1.2 \mathrm{~A}+0.84 \mathrm{~V} 1)$} \\
\hline \multirow{2}{*}{ Pavimento } & \multicolumn{2}{|c|}{ Desloc. Horizontal (cm) } & \multirow{2}{*}{ Pavimento } & \multicolumn{2}{|c|}{ Desloc. Horizontal $(\mathrm{cm})$} & \multirow{2}{*}{ Pavimento } & \multicolumn{2}{|c|}{ Desloc. Horizontal $(\mathrm{cm})$} \\
\hline & Eixo $X$ & Eixo $Y$ & & Eixo $X$ & Eixo $Y$ & & Eixo $X$ & Eixo $Y$ \\
\hline cobertura & 0.40 & 0.13 & Cobertura & 0.41 & 0.12 & cobertura & 0.36 & 0.12 \\
\hline pavimento tipo 4 & 0.36 & 0.11 & Pavimento tipo 4 & 0.36 & 0.09 & pavimento tipo 4 & 0.33 & 0.09 \\
\hline Pavimento tipo 3 & 0.30 & 0.09 & Pavimento tipo 3 & 0.30 & 0.06 & Pavimento tipo 3 & 0.27 & 0.06 \\
\hline Pavimento tipo 2 & 0.21 & 0.06 & Pavimento tipo 2 & 0.21 & 0.04 & Pavimento tipo 2 & 0.20 & 0.04 \\
\hline Pavimento tipo 1 & 0.11 & 0.02 & Pavimento tipo 1 & 0.11 & 0.02 & Pavimento tipo 1 & 0.10 & 0.02 \\
\hline fundação & 0.02 & 0.00 & Fundação & 0.02 & 0.00 & fundação & 0.02 & 0.00 \\
\hline \multicolumn{3}{|c|}{ Eixo $Y(1.3 \mathrm{G} 1+1.4 \mathrm{G} 2+1.4 \mathrm{~S}+1.4 \mathrm{Q}+1.2 \mathrm{~A}+0.84 \mathrm{~V} 3)$} & \multicolumn{3}{|c|}{ Eixo $Y(1.3 \mathrm{G} 1+1.4 \mathrm{G} 2+1.4 \mathrm{~S}+1.4 \mathrm{Q}+1.2 \mathrm{~A}+0.84 \mathrm{~V} 3)$} & \multicolumn{3}{|c|}{ Eixo $Y(1.3 \mathrm{G} 1+1.4 \mathrm{G} 2+1.4 \mathrm{~S}+1.4 \mathrm{Q}+1.2 \mathrm{~A}+0.84 \mathrm{~V} 3)$} \\
\hline cobertura & 0.03 & 0.77 & Cobertura & 0.05 & 0.71 & cobertura & 0.00 & 0.71 \\
\hline pavimento tipo 4 & 0.02 & 0.71 & Pavimento tipo 4 & 0.04 & 0.64 & pavimento tipo 4 & 0.00 & 0.63 \\
\hline Pavimento tipo 3 & 0.01 & 0.59 & Pavimento tipo 3 & 0.03 & 0.52 & Pavimento tipo 3 & 0.00 & 0.52 \\
\hline Pavimento tipo 2 & 0.01 & 0.42 & Pavimento tipo 2 & 0.02 & 0.37 & Pavimento tipo 2 & 0.00 & 0.37 \\
\hline Pavimento tipo 1 & 0.00 & 0.21 & Pavimento tipo 1 & 0.01 & 0.19 & Pavimento tipo 1 & 0.00 & 0.19 \\
\hline fundação & 0.00 & 0.02 & Fundação & 0.00 & 0.03 & fundação & 0.00 & 0.03 \\
\hline
\end{tabular}

De acordo com a NBR 6118 (ABNT, 2014) com os valores de Gama-Z obtidos pode-se concluir que se trata de uma estrutura de nós fixos ou indeslocáveis, visto que esses valores não ultrapassaram 1,10, conforme a figura 3.
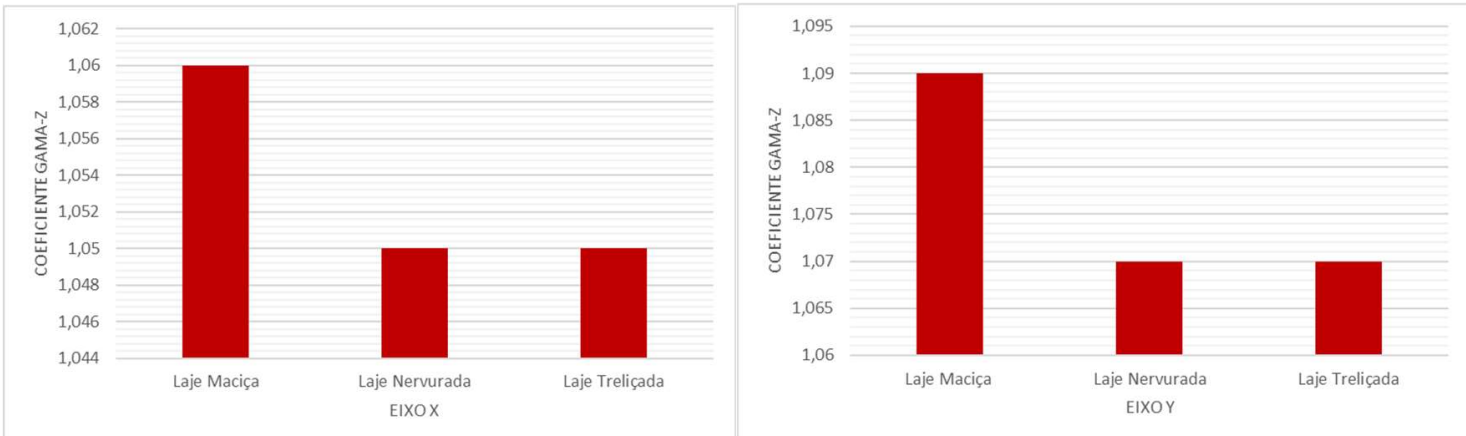

Figura 3: Variação do Gama-Z para as direções x e y. 


\section{CONCLUSÕES}

É muito relevante o estudo comparativo entre os métodos construtivos existentes. Percebeu-se que a principal vantagem da laje maciça, bastante utilizada em construções de pequeno a médio porte, consiste no fato de ela apresentar alto grau de resistência a trincas e fissuras; como desvantagens tem-se a geração de muitos resíduos devido à alta quantidade de material utilizado nas formas, maior peso e, de maneira geral, um custo financeiro elevado. As lajes nervuradas apresentam como vantagens maior economia, vence grandes vãos, maior liberdade arquitetônica; desvantagens: dificuldade de compatibilização com outras etapas de projeto e exige mão de obra especializada. As lajes treliçadas têm como vantagens o fato de serem mais leves, rapidez na execução, não exige mão de obra especializada, ajuda no isolamento térmico, baixo custo; desvantagens: dependendo do tipo de materiais utilizados, a execução torna-se complexa.

Analisando os resultados obtidos pode-se notar que, em todos os métodos o Gama-Z encontrado possui valores bem semelhantes, os das lajes nervuradas e treliçadas inclusive foram idênticos, porém, todos menores que 1,10 o que permite concluir que a estrutura é de nós fixos ou indeslocáveis. Ressalta-se ainda que, na verdade, entre esses métodos construtivos estudados, não há um superior ao outro. Isto é, o método mais adequado dependerá muito das especificações do cliente, a disponibilidade dos materiais no mercado, entre outros fatores.

\section{REFERÊNCIAS}

ABNT. Associação Brasileira de Normas Técnicas. NBR 6118: projeto de estruturas de concreto - procedimento. Rio de Janeiro: ABNT, 2014.
ABNT. Associação Brasileira de Normas Técnicas. NBR-6123: Forças devidas ao vento em edificações. Rio de Janeiro: ABNT, 1988.

PINHEIRO, L. M.. Notas de aula da disciplina Estruturas de Concreto A. São Carlos: EESC-USP, 2003.

A CBPC - Companhia Brasileira de Produção Científica (CNPJ: 11.221.422/0001-03) detém os direitos materiais desta publicação. Os direitos referem-se à publicação do trabalho em qualquer parte do mundo, incluindo os direitos às renovações, expansões e disseminações da contribuição, bem como outros direitos subsidiários. Todos os trabalhos publicados eletronicamente poderão posteriormente ser publicados em coletâneas impressas sob coordenação da Sapientiae Publishing, da Companhia Brasileira de Produção Científica e seus parceiros autorizados. Os (as) autores (as) preservam os direitos autorais, mas não têm permissão para a publicação da contribuição em outro meio, impresso ou digital, em português ou em tradução. 